\title{
Signaling in large-scale neural networks
}

\author{
Rune W. Berg · Jørn Hounsgaard
}

Received: 11 April 2008/Revised: 15 October 2008/Accepted: 16 October 2008/Published online: 14 November 2008

(C) The Author(s) 2008. This article is published with open access at Springerlink.com

\begin{abstract}
We examine the recent finding that neurons in spinal motor circuits enter a high conductance state during functional network activity. The underlying concomitant increase in random inhibitory and excitatory synaptic activity leads to stochastic signal processing. The possible advantages of this metabolically costly organization are analyzed by comparing with synaptically less intense networks driven by the intrinsic response properties of the network neurons.
\end{abstract}

\section{Introduction}

It seems that behavioral diversity and flexibility scale with the number of interconnected neurons in nervous systems. For this reason alone, the relation between behavior and the properties and activity of individual neurons becomes increasingly intractable in larger brains. Furthermore, recent decades have shown that the response properties of neurons are dynamic, highly non-linear and unique for each cell type in the brain. A general theory on how to bridge the gap between cell signaling at the microscopic level and function at the macroscopic level in large-scale networks is not in sight. Here, we explore basic questions that may guide

\section{R. W. Berg · J. Hounsgaard}

Department of Neuroscience and Pharmacology, University of Copenhagen, Blegdamsvej 3, 2200 Copenhagen N, Denmark URL: http://www.mfi.ku.dk/hounsgaard-lab/

J. Hounsgaard $(\square)$

INF, The PANUM Institute, Bldg. 12-5-9, University

of Copenhagen, Blegdamsvej 3, 2200 Copenhagen N, Denmark

e-mail: j.hounsgaard@mfi.ku.dk

URL: http://www.mfi.ku.dk/hounsgaard-lab/ diagnostic experimentation aimed at the formation of theoretical insights beyond case by case numerical modeling.

Sensory afferents and motor efferents provide strategic anchors for experimentation on nervous systems because their activity is uniquely decodable by means of external sensory and motor reference frames. For this reason, our focus is on networks that perform sensory-motor transduction. The possible function and relative contribution of synaptic and intrinsic conductances to cell firing during functional network activity will figure in the discussion of each of the questions posed.

\section{The neuronal coding problem}

Sensory-motor transduction is a fundamental process in living organisms from protozoan eukaryotes and onwards (Banchetti 2005). Motor behavior probably constituted the key evolutionary drive for the differentiation of nervous systems (Llinás 2000). Early nerve cells may have emerged as isolated multifunctional sensory, motor and secretory 'allin-one' cells derived from the epithelium in Cnidarians more than 700 millions years ago (Lentz 1968; Miljkovic-Licina et al. 2004; Westfall 1996). From these origins, interneurons evolved to become mediators between specific sensory cells and specialized effector cells such as nematocysts and muscles. Organized in non-polarized nerve nets, interneurons could mediate integrative sensing of environmental information so that appropriate behaviors followed (Satterlie and Nolen 2001). However, in becoming abstract signaling devices, the interneurons also gave rise to the fundamental coding problem still inherent in the study of nervous systems: information is carried and processed by molecular machineries in individual nerve cells while function is the collective sum of the activity of interacting neurons organized in 
networks. The gap between the constituents and the functional whole is aggravated in large-scale networks because neurons receive signals from a large number of other neurons. For this reason, the activity of individual neurons is rarely directly relatable to singular events in other neurons or in the outside world. Because neurons process synaptic input and reduce information, it is impossible to reconstruct their input patterns entirely from their output. In addition, it is practically never possible to record all the presynaptic input patterns that give rise to particular output in a neuron. Even worse, the information coded by these input patterns will usually not themselves be decodable. For all these reasons the coding that real neurons perform is not well understood. In fact, the relation between signaling and coding in neurons and function at the network level is one of the hard problems in neuroscience. Nevertheless, there is much to be said about synaptic transduction in individual neurons. Intercalated between the synaptic currents and spike generation are active non-linear filters provided by current generating voltage gated ion channels (Llinas 1988). Since nerve cells differ in the kind, density and distribution of the ion channels that they express, they also differ in the way incoming information is processed to spike patterns. Awareness of this active filter function and its potential significance spurred a flurry of experimentation to characterize the non-linear properties, their underlying mechanisms and functions in neurons in a range of networks and organisms (Llinas 1988; London and Hausser 2005; Selverston and Moulins 1985; ToledoRodriguez et al. 2005). The intrinsic response properties were seen to provide a bias that favored certain spike patterns over others, particularly time varying activity like oscillations (Llinas 1988). The mix of voltage sensitive ion channels in each cell would be well suited to reduce the problem of pattern formation by funneling a wide range of noisy synaptic input into a few pre-selected 'useful' output patterns provided by the intrinsic response properties (Hounsgaard and Midtgaard 1989; Midtgaard 1989). The role of intrinsic response properties of individual neurons in the formation of 'useful' activity patterns in networks of neurons is well supported by experimental evidence from oligo-cellular circuits with the stomastogastric ganglion as a prime example (Marder and Bucher 2007). Even in larger functional circuits intrinsic response properties of neurons are thought to play a key role in formation of time scale, dynamics and coherence (Grillner 2003; Llinas 1988).

\section{Cell properties and irregular firing}

We have seen that neurons in all nervous systems are equipped with non-linear intrinsic response properties. Gated by voltage the underlying ion channels interact to produce dynamic trans-membrane currents. The resulting variations in membrane potential are continuous in time, smoothened by the large number of contributing channels and by membrane capacitance. Therefore, perturbations in trans-membrane current in isolated neurons will produce smooth changes in membrane potential and firing rate. However, during behavior neurons often display highly irregular firing rates (Knierim and van Essen 1992; Newsome et al. 1989; Zoccolan et al. 2002). Firing with a high coefficient of variation is not easily accounted for by a smoothly varying membrane potential or by integration of excitatory synaptic potentials (Koch 1999; Softky and Koch 1993). On the other hand, uncorrelated interspike intervals automatically emerge from 'noisy' fluctuations in membrane potential (Calvin and Stevens 1967) that readily arise from randomly elicited excitatory and inhibitory synaptic potentials (Gerstein and Mandelbrot 1964; Softky and Koch 1993). Behavioral states in which neurons receive mixed inhibitory and excitatory signals have now been reported in the cerebral cortex (Marino et al. 2005; Steriade et al. 2001) and in the spinal cord (Berg et al. 2007). Concurrent increase in inhibition and excitation leads to a dramatic increase in average conductance even for a moderate depolarization from the resting membrane potential (Berg et al. 2007; Destexhe et al. 2003; Haider et al. 2006; Marino et al. 2005). High conductance states by balanced increase in inhibition and excitation are characterized by a dramatic increase in the amplitude and power spectrum of membrane potential fluctuations due to high density of uncorrelated inhibitory and excitatory synaptic conductances (Berg et al. 2007). It is these fluctuations that lead to irregular firing rates. Further support for a widespread occurrence of high conductance states during functional network activity is their association with the UPstates proposed to be the active state of neurons during behavior (Destexhe et al. 2007, 2003; Haider et al. 2006).

\section{Current and conductance-based circuits}

The essentials of network dynamics are synaptic interactions between the constituent neurons. Synaptic input in neurons can be modeled as synaptic current or synaptic conductance (for a review see Burkitt 2006). The resulting network models are generally referred to as current based and conductance based, respectively. In the latter case, the change in driving force and input resistance is taken into account, while these are assumed constant in the current-based network. Explicitly written the dynamical equations for the membrane potential in a one-compartment model are:

Current-based synapses:

$C \frac{\mathrm{d} V_{m}}{\mathrm{~d} t}=\sum_{j} G_{j} \cdot\left(E_{j}-V_{m}\right)+I_{\text {Syn }}$

where $I_{\text {syn }}$ refers to the average net synaptic current. Conductance-based synapses: 


$$
\begin{aligned}
C \frac{\mathrm{d} V_{m}}{\mathrm{~d} t}= & \sum_{j} G_{j} \cdot\left(E_{j}-V_{m}\right)+G_{\mathrm{I}} \cdot\left(E_{\mathrm{I}}-V_{m}\right) \\
& +G_{\mathrm{E}} \cdot\left(E_{\mathrm{E}}-V_{m}\right)
\end{aligned}
$$

where the $G_{j}$ refer to the intrinsic conductance of a membrane channels and the $E_{j} s$ are their respective reversal potentials. The $G_{\mathrm{I}}$ and $G_{\mathrm{E}}$ are the total inhibitory and excitatory synaptic conductance and $E_{\mathrm{I}}$ and $E_{\mathrm{E}}$ are their respective reversal potentials.

In the current-based synapse, the synaptic current is assumed independent on both changes in total conductance and driving force, whereas in the conductance-based synapse both of these are taken into account. The difference may seem subtle, but under certain circumstances the distinction is essential (see e.g., Kuhn et al. 2004). The current-based description is widely used because of its simplicity and its justified application in weakly connected networks. In networks with high intensity of synaptic activity, however, a conductance-based description is necessary. In the following, we describe the two network modes. (1) In quiescent networks, i.e., networks with low levels of synaptic activity, pattern generating neurons interact like mechanical oscillators weakly interconnected with springs (Berg et al. 2008). Inhibitory and excitatory synaptic connections entrain neurons in network rhythms during which individual cells fire with regular rates (Fig. 1a). In computer models, networks of this kind readily perform stable rhythmic activity as shown by a wide range of simulated motor networks. Rhythms can be modified by modulation of the ion channels underlying the intrinsic response properties of constituent neurons (Grillner 2003; Marder and Bucher 2007). Intuitive attributes of networks with low synaptic intensity are energy efficiency due to low conductance increase during activity (Attwell and Gibb 2005; Attwell and Laughlin 2001) and ability to transform brief synaptic currents to voltage changes on multiple time scales (Hounsgaard and Midtgaard 1989). Simulated networks of this kind are expressed in terms of coupled differential equations. (2) In contrast, networks of intense activity of inhibitory and excitatory neurons are dominated by synaptic conductances (Fig. 1b). By domination, we mean when the synaptic conductance is large compared with the slow intrinsic conductances, the membrane potential is "dominated" by synaptic activity. Let us write this explicitly from the generic membrane equation:
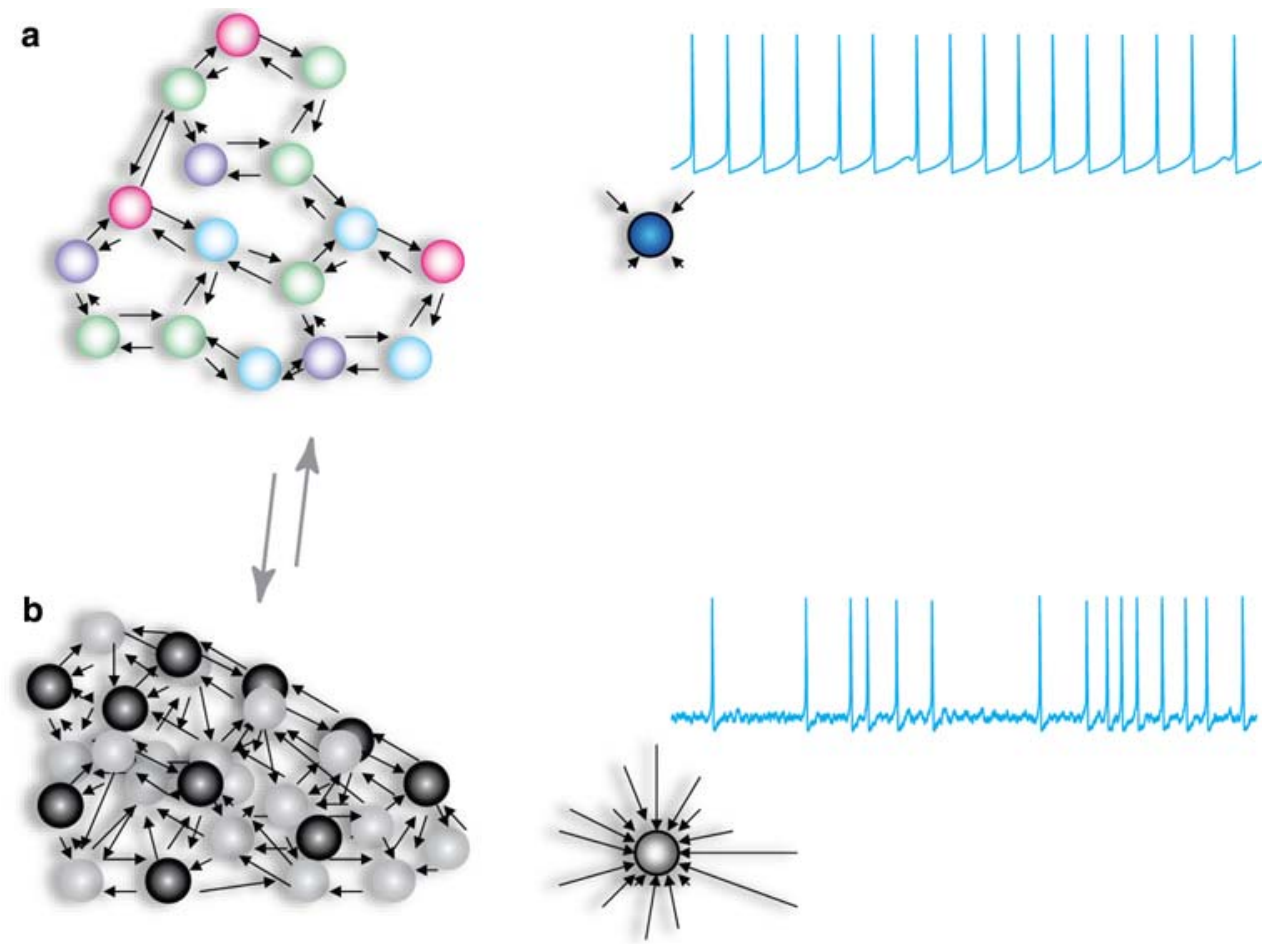

Fig. 1 Neurons in two types of networks. a. Low-intensity network: left, few active neurons result in low synaptic conductance and therefore the neurons express a range of complex intrinsic response properties as indicated by colored cell bodies. Right, typical activity pattern of a single neuron embedded in such a network. Synaptic input is of low intensity and spiking regular and largely determined by the intrinsic properties of the cell itself. b. High intensity network: the inhibitory and excitatory neurons (black and gray) project to many other neurons and receive intense stochastic input (Left). Right: the activity of a typical neuron is irregular due to the fluctuating intense synaptic input. This intense input causes a substantial decrease in input resistance and therefore shunts the slow intrinsic properties so the different cells diminish their individuality (illustrated as lack of color of cell body) 


$$
\begin{aligned}
C \frac{\mathrm{d} V_{m}}{\mathrm{~d} t}= & \sum_{j} G_{j} \cdot\left(E_{j}-V_{m}\right)+G_{\text {syn }} \cdot\left(E_{\text {syn }}-V_{m}\right) \\
& +G_{\text {leak }} \cdot\left(E_{\text {leak }}-V_{m}\right)
\end{aligned}
$$

the total conductance is the sum of the parts:

$G_{\text {total }}=G_{\text {leak }}+\sum_{j} G_{j}+G_{\text {syn }}$

When the membrane potential has reached steady state, its value is given by the average of the reversal potentials weighted by their individual conductances:

$V_{m}=\sum_{j} \frac{G_{j}}{G_{\text {total }}} E_{j}+\frac{G_{\text {leak }}}{G_{\text {total }}} E_{\text {leak }}+\frac{G_{\text {syn }}}{G_{\text {total }}} E_{\text {syn }}$

However, when the neuron receives high intensity of synaptic activity and it is in "high conductance" state, the synaptic conductance is dominating, i.e.,

$G_{\text {syn }}>>\sum_{j} G_{j}$

and the average can be approximated to

$V_{m} \approx \frac{G_{\text {leak }}}{G_{\text {total }}} E_{\text {leak }}+\frac{G_{\text {syn }}}{G_{\text {total }}} E_{\text {syn }}$

The activity patterns are determined by network structure, including the density and distribution of synaptic connections. During network activity, neurons fire with irregular rates determined by the fluctuating membrane potential (Berg et al. 2008). Attributes of densely connected balanced networks are high metabolic cost, fast response times and high temporal resolution (van Vreeswijk and Sompolinsky 1996; Berg et al. 2008) accompanied by chaotic states with weak spatial correlations (van Vreeswijk and Sompolinsky 1998). Simulated networks of this kind are expressed in terms of stochastic variables and probabilites (Destexhe and Contreras 2006).

High conductance states in large-scale spinal networks

Data on cell firing, membrane conductance and power of synaptic fluctuations in membrane potential during functional network activity is extremely sparse, coming from just a few cell types in a handful of networks. For this reason, it is not possible to assess the prevalence of current and conductance-based regimes during functional network activity. It is quite likely that the same neuron can operate in a mode that can be closely approximated as current based in some situations, whereas in other situations a conductance-based regime is necessary, depending on the behavior in which the network is engaged in (Berkowitz 2005). The high conductance state produced by parallel increase in inhibitory and excitatory synaptic activity is energetically costly. Their mere existence suggests that such states are valuable in the functional network activity that they are part of. We will end by contemplating what these advantages might be.

1. Network reliability. By having many intensely interacting neurons, each individual neuron makes an insignificant contribution. This implies a reliable probabilistic type of coding that is distributed over many neurons (Shadlen and Newsome 1998). One of the consequences of this large population coding is that the total synaptic conductance becomes high and stochastic on short time scales.

2. Gain control. Modulation of the intensity of synaptic input in a balanced manner change the input-output response characteristics of neurons (Chance et al. 2002). This may be useful in spinal circuits and for motoneurons in particular. It is currently a puzzle how the large precision of movements in different dynamical contexts is achieved. Controlling the motoneuron gain and spinal circuit gain via intensity of balanced inhibition and excitation may add precision to skilled movements (Berg and Hounsgaard 2007).

3. Increased temporal agility. The high conductance state substantially reduce the input resistance and sharpen the temporal processing several fold (Berg et al. 2008; Barrett 1975; Bernander et al. 1991). Having a fast motor system is essential for matching the rapid sensory processing. Currently, there is no convincing theory on how speed and agility is achieved in the spinal motor circuit except by reducing the input resistance by balancing excitation with inhibition (Berg et al. 2008).

4. Dendritic integration. Spinal neurons and motoneurons in particular, have extensive dendritic trees which intuitively may seem useless in a high conductance state where the electrotonic distance is severely decreased. However, the high conductance state also increase the fluctuations of the membrane potential in dendritic branches, which could provide stochastic resonance in concert with distal excitable properties and thus ensure transmission of certain dendritic potentials (Rudolph and Destexhe 2003).

5. Activity control. The concurrent increase inhibition and excitation may be essential to maintain stability in the active spinal network. The occurrence of seizures in response to even small reductions in inhibition emphasizes that a remarkable fine balance is necessary, to avoid a runaway excitation.

We will continue to use the spinal sensory motor network underlying scratch behavior in turtles as our experimental reference system (Stein 2005). Hindlimb scratching is the goal-directed motor act in which a peripheral part of the limb is repeatedly rubbed against 
the site on the body where the eliciting mechanical stimulus was applied. It is in this model that we have seen motoneurons and interneurons enter the high conductance state due to a parallel increase in excitatory and inhibitory synaptic activity during scratching (Alaburda et al. 2005; Berg et al. 2007). This was a surprising finding for two reasons. First, prior examples of high conductance states had all been seen in the cerebral cortex and the basal ganglia (Destexhe et al. 2003). They were thought to be mechanisms added on late in evolution to support higher brain functions and were encountered in sensory and cognitive network activity. Secondly, neurons in the spinal motor system are equipped with intricate intrinsic properties that would seem ideal to support network activity on the time scale of locomotor and scratch behavior (Delgado-Lezama and Hounsgaard 1999; Grillner 2003; Russo and Hounsgaard 1999). Furthermore, these properties are regulated by intrinsic and extrinsic modulation, again, seemingly ideal for dynamic adjustments and external controls (Alaburda and Hounsgaard 2003; Russo and Hounsgaard 1999). With flexible modulation of intrinsic properties and synaptic strengths, current-based networks can be shifted over a considerable range (Grillner 2003; Hooper and Moulins 1989; Hounsgaard and Midtgaard 1989) and even be broken up in independent sub-networks (Hooper and Moulins 1989; Simmers et al. 1995). With emergence of limbed vertebrates; however, the requirement for flexibility increased by orders of magnitude. The sequence and strength of contractions of different muscle groups can be rearranged in innumerous ways from moment to moment, e.g., when shifting between walking, kicking and dancing. Are high conductance networks helpful in achieving such flexibility?

Specification. The first problem to consider is how a sensory input might generate a network state that leads to the appropriate behavior, here scratching. For the hindlimb rostral scratch it is known that primary afferents activated by the mechanical stimulus activates an isolated second order sensory network several segments rostral to scratch motor network (Currie and Stein 1990). Cells in this network display long-lasting activity in response a brief mechanical stimulus. We speculate that this sustained network activity could act not only as tonic drive for the lumbar motor network but might also be wired up with the lumbar motor network so that the drive also brings the motor network in 'rostral scratch mode' by targeted ionotropic and metabotropic synaptic mechanisms. It is reasonable to assume that similar isolated driver networks are activated in the segments with projections from the receptive fields for the caudal and pocket scratch forms (Stein 2005).

\section{Confinement}

The firewall hypothesis For each motor behavior, a particular sub-network has to be 'carved out' of an anatomically continuous spinal network so that activity is confined to the relevant motor pools. We propose that 'confinement' in the balanced state is achieved by a 'firewall effect' surrounding the active network. The neurons in the functional network are active because the parallel increase in inhibitory and excitatory activity brings the average membrane potential from a hyperpolarized low conductance level to a high conductance level near threshold for action potentials. These active neurons are surrounded by neurons in which the balanced increase in inhibitory and excitatory activity is less intense. Such neurons will depolarize less and the synaptically induced fluctuations in membrane potential will not reach threshold. This 'subliminal fringe' of neurons could act as a fire-wall to prevent the active network activity from spreading and at the same time make synaptic 'break-in' from the outside difficult due to the shunting effect of the high conductance state.

\section{Origin of time scale}

In oscillator models dynamics come directly from the kinetics of channels and synaptic interactions. At present it is not clear if varied temporal dynamics in balanced networks can emerge from synaptic interactions alone or require additional mechanisms like synaptic habituation or accumulating changes in membrane potential. In the case of the scratch rhythm the fundamental question is if the scratch period is defined by the dynamics of the constituent neurons or emerges from distributed synaptic interactions or by a combination as proposed for respiration (Feldman and Del Negro 2006). This would entail that the rhythm is the result of self-organization of the large-scale network activity. In such a situation the time scale of the network rhythm does not have to be reflected in the time scale of the activity of its neurons in isolation. This type of macroscopic slow time scales have been observed in other selforganizing biological systems, such as nest building by ants, honey combs building by bees, and mounds by termites to mention some (Camazine et al. 2001). The time scale of activity of its constituents (ants, bees, and termites) is much faster than the macroscopic outcome (the nest, comb, and mounds). In a similar fashion the spinal motor rhythms could be a slow emergent network property of the much faster activity of the constituent neurons. Although this analogy is appealing, it remains to be molded into a concrete theory and investigated experimentally.

In summary, we have presented a dilemma in the approach to large-scale functional networks of neurons. If 
their modus operandi is based on weakly coupled neurons with non-linear intrinsic response properties, then it is necessary to characterize these properties in the constituent cell types in each network and reveal how they respond to naturally occurring synaptic input patterns. It is equally important to unravel the anatomical connectivity. On the other hand, in the case of high conductance states the global mechanisms of network activity should be approached from the statistical nature of the fluctuations in membrane conductance, membrane potential and firing rate in individual cells during functional network activity. The circuit architecture that leads to parallel increase in inhibitory and excitatory synaptic activity must be identified (Miura et al. 2007). In addition, major focus should be on identifying the avalanche that leads from particular external input to the appropriate behavior, in our case how a light brush on the carapace generates a network state for the appropriate scratch form. What are the network mechanisms that stabilize a few distinct behavioral modes in a continuous large-scale network and secure their differential selection by external synaptic input?

The experimental resolution of these dilemmas is crucial for the development of more specific understanding of the relation between signal processing at the cellular level and circuit function in large-scale networks.

Open Access This article is distributed under the terms of the Creative Commons Attribution Noncommercial License which permits any noncommercial use, distribution, and reproduction in any medium, provided the original author(s) and source are credited.

\section{References}

Alaburda A, Hounsgaard J (2003) Metabotropic modulation of motoneurons by scratch-like spinal network activity. J Neurosci 23:8625-8629

Alaburda A, Russo R, MacAulay N, Hounsgaard J (2005) Periodic high-conductance states in spinal neurons during scratch-like network activity in adult turtles. J Neurosci 25:6316-6321. doi: 10.1523/JNEUROSCI.0843-05.2005

Attwell D, Gibb A (2005) Neuroenergetics and the kinetic design of excitatory synapses. Nat Rev Neurosci 6:841-849. doi: 10.1038/nrn1784

Attwell D, Laughlin SB (2001) An energy budget for signaling in the grey matter of the brain. J Cereb Blood Flow Metab 21:11331145. doi:10.1097/00004647-200110000-00001

Banchetti R (2005) From the ethology of unicellular eukaryotes to the locomotion of the living beings: meaning and evolution of the phenomenon. Riv Biol 98:111-123

Barrett JN (1975) Motoneuron dendrites: role in synaptic integration. Fed Proc 34:1398-1407

Berg RW, Hounsgaard J (2007) Balanced inhibition and excitation: a mechanism for gain modulation in spinal motoneurons? Soc Neurosci Abstracts 34:408.4

Berg RW, Alaburda A, Hounsgaard J (2007) Balanced inhibition and excitation drive spike activity in spinal half-centers. Science 315:390-393. doi:10.1126/science.1134960
Berg RW, Ditlevsen S, Hounsgaard J (2008) Intense synaptic activity enhances temporal resolution in spinal motoneurons. PLoS ONE 3(9):e3218. doi:10.1371/journal.pone.0003218

Berkowitz A (2005) Physiology and morphology indicate that individual spinal interneurons contribute to diverse limb movements. J Neurophysiol 94:4455-4470. doi:10.1152/jn.00229. 2005

Bernander O, Douglas RJ, Martin KA, Koch C (1991) Synaptic background activity influences spatiotemporal integration in single pyramidal cells. Proc Natl Acad Sci USA 88:1156911573. doi:10.1073/pnas.88.24.11569

Burkitt AN (2006) A review of the integrate-and-fire neuron model: 1. homogeneous synaptic input. Biol Cybern 95:1-19. doi:10.1007/ s00422-006-0068-6

Calvin WH, Stevens CF (1967) Synaptic noise as a source of variability in the interval between action potentials. Science 155:842-844. doi:10.1126/science.155.3764.842

Camazine S, Deneubourg J-L, Franks NR, Sneyd J, Theraulaz G, Bonabeau E (2001) Self-organization in biological systems. Princeton University Press, Princeton, NJ

Chance FS, Abbott LF, Reyes AD (2002) Gain modulation from background synaptic input. Neuron 35:773-782. doi:10.1016/ S0896-6273(02)00820-6

Currie SN, Stein PS (1990) Cutaneous stimulation evokes long-lasting excitation of spinal interneurons in the turtle. J Neurophysiol 64:1134-1148

Delgado-Lezama R, Hounsgaard J (1999) Adapting motoneurons for motor behavior. Prog Brain Res 123:57-63. doi:10.1016/S00796123(08)62844-7

Destexhe A, Contreras D (2006) Neuronal computations with stochastic network states. Science 314:85-90. doi:10.1126/ science. 1127241

Destexhe A, Rudolph M, Pare D (2003) The high-conductance state of neocortical neurons in vivo. Nat Rev Neurosci 4:739-751. doi:10.1038/nrn1198

Destexhe A, Hughes SW, Rudolph M, Crunelli V (2007) Are corticothalamic 'up' states fragments of wakefulness? Trends Neurosci 30:334-342. doi:10.1016/j.tins.2007.04.006

Feldman JL, Del Negro CA (2006) Looking for inspiration: new perspectives on respiratory rhythm. Nat Rev Neurosci 7:232242. doi: $10.1038 / \mathrm{nrn} 1871$

Gerstein GL, Mandelbrot B (1964) Random walk models for the spike activity of a single neuron. Biophys J 4:41-68

Grillner S (2003) The motor infrastructure: from ion channels to neuronal networks. Nat Rev Neurosci 4:573-586. doi:10.1038/ $\operatorname{nrn} 1137$

Haider B, Duque A, Hasenstaub AR, McCormick DA (2006) Neocortical network activity in vivo is generated through a dynamic balance of excitation and inhibition. J Neurosci 26:4535-4545. doi:10.1523/JNEUROSCI.5297-05.2006

Hooper SL, Moulins M (1989) Switching of a neuron from one network to another by sensory-induced changes in membrane properties. Science 244:1587-1589. doi:10.1126/science. 2740903

Hounsgaard J, Midtgaard J (1989) Dendrite processing in more ways than one. Trends Neurosci 12:313-315. doi:10.1016/0166-2236 (89)90036-2

Knierim JJ, van Essen DC (1992) Neuronal responses to static texture patterns in area V1 of the alert macaque monkey. J Neurophysiol 67:961-980

Koch C (1999) Biophysics of computation: information processing in single neurons. Oxford University Press, New York

Kuhn A, Aertsen A, Rotter S (2004) Neuronal integration of synaptic input in the fluctuation-driven regime. J Neurosci 24(10):23452356. doi:10.1523/JNEUROSCI.3349-03.2004 
Lentz TL (1968) Primitive nervous systems. Yale University Press, New Haven

Llinás R (2000) I of the vortex: from neurons to self. The MIT Press, Cambridge

Llinas RR (1988) The intrinsic electrophysiological properties of mammalian neurons: insights into central nervous system function. Science 242:1654-1664. doi:10.1126/science.3059497

London M, Hausser M (2005) Dendritic computation. Annu Rev Neurosci 28:503-532. doi:10.1146/annurev.neuro.28.061604. 135703

Marder E, Bucher D (2007) Understanding circuit dynamics using the stomatogastric nervous system of lobsters and crabs. Annu Rev Physiol 69:291-316. doi:10.1146/annurev.physiol.69.031905. 161516

Marino J, Schummers J, Lyon DC, Schwabe L, Beck O, Wiesing P, Obermayer K, Sur M (2005) Invariant computations in local cortical networks with balanced excitation and inhibition. Nat Neurosci 8:194-201. doi:10.1038/nn1391

Midtgaard JHJ (1989) Nerve cells as source of time scale and processing density in brain function. IJNS Int J Neural Syst $1: 89-94$

Miljkovic-Licina M, Gauchat D, Galliot B (2004) Neuronal evolution: analysis of regulatory genes in a first-evolved nervous system, the hydra nervous system. Biosystems 76:75-87. doi:10.1016/j. biosystems.2004.05.030

Miura K, Tsubo Y, Okada M, Fukai T (2007) Balanced excitatory and inhibitory inputs to cortical neurons decouple firing irregularity from rate modulations. $\mathrm{J}$ Neurosci 27:13802-13812. doi:10.1523/JNEUROSCI.2452-07.2007

Newsome WT, Britten KH, Movshon JA (1989) Neuronal correlates of a perceptual decision. Nature 341:52-54. doi:10.1038/ $341052 \mathrm{a} 0$

Rudolph M, Destexhe A (2003) A fast-conducting, stochastic integrative mode for neocortical neurons in vivo. $\mathrm{J}$ Neurosci 23:2466-2476

Russo RE, Hounsgaard J (1999) Dynamics of intrinsic electrophysiological properties in spinal cord neurones. Prog Biophys Mol Biol 72:329-365. doi:10.1016/S0079-6107(99)00011-5
Satterlie RA, Nolen TG (2001) Why do cubomedusae have only four swim pacemakers? J Exp Biol 204:1413-1419

Selverston AI, Moulins M (1985) Oscillatory neural networks. Annu Rev Physiol 47:29-48. doi:10.1146/annurev.ph.47.030185. 000333

Shadlen MN, Newsome WT (1998) The variable discharge of cortical neurons: implications for connectivity, computation, and information coding. J Neurosci 18:3870-3896

Simmers J, Meyrand P, Moulins M (1995) Modulation and dynamic specification of motor rhythm-generating circuits in crustacea. J Physiol (Paris) 89:195-208. doi:10.1016/0928-4257(96)83636-9

Softky WR, Koch C (1993) The highly irregular firing of cortical cells is inconsistent with temporal integration of random EPSPs. J Neurosci 13:334-350

Stein PS (2005) Neuronal control of turtle hindlimb motor rhythms. J Comp Physiol A Neuroethol Sens Neural Behav Physiol 191:213-229

Steriade M, Timofeev I, Grenier F (2001) Natural waking and sleep states: a view from inside neocortical neurons. J Neurophysiol 85:1969-1985

Toledo-Rodriguez M, El Manira A, Wallen P, Svirskis G, Hounsgaard J (2005) Cellular signalling properties in microcircuits. Trends Neurosci 28:534-540. doi:10.1016/j.tins.2005.08.001

van Vreeswijk C, Sompolinsky H (1996) Chaos in neuronal networks with balanced excitatory and inhibitory activity. Science 274:1724-1726. doi:10.1126/science.274.5293.1724

van Vreeswijk C, Sompolinsky H (1998) Chaotic balanced state in a model of cortical circuits. Neural Comput 10:1321-1371. doi: $10.1162 / 089976698300017214$

Westfall J (1996) Ultrastructure of synapses in the first-evolved nervous systems. J Neurocytol 25:735-746. doi:10.1007/ BF02284838

Zoccolan D, Pinato G, Torre V (2002) Highly variable spike trains underlie reproducible sensorimotor responses in the medicinal leech. J Neurosci 22:10790-10800 